\title{
BIM-Enabled Issue and Progress Tracking Services Using Mixed Reality
}

\section{Conference Paper}

\section{Author(s):}

Holzwarth, Valentin; Steiner, Sebastian; Schneider, Johannes; vom Brocke, Jan; Kunz, Andreas (D)

Publication date:

2021-06-26

Permanent link:

https://doi.org/10.3929/ethz-b-000492593

Rights / license:

In Copyright - Non-Commercial Use Permitted

Originally published in:

Progress in IS, https://doi.org/10.1007/978-3-030-72090-2_5 


\title{
BIM-Enabled Issue and Progress Tracking Services Using Mixed Reality
}

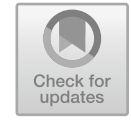

\author{
Valentin Holzwarth, Sebastian Steiner, Johannes Schneider, Jan vom Brocke, \\ and Andreas Kunz
}

\begin{abstract}
This paper reports on a case study in collaboration with an industry partner to explore the value creation potentials of Mixed Reality (MR) in construction. MR is a technology that allows for intuitive interaction with digital data through overlays on the real world, giving immediate access to the data of a construction site to quickly track or resolve possible issues. While prior research identified potential applications of MR in construction, the evaluation of MR in real contexts is lacking. Thus, this paper describes a user study that evaluates an MR-assisted inspection task with the participation of nine construction professionals. The results reveal that the average time needed to track an issue with the Building Information Modeling (BIM)-enabled MR application takes less than a minute, compared to an hour for the standard process. Furthermore, the participants reported that working with the MR application was enjoyable and they would like to use it more frequently. Finally, based on the user study's findings, this paper develops two service concept prototypes that could be implemented by the industry partner.
\end{abstract}

Keywords Mixed reality $\cdot$ AEC $\cdot$ Building information modeling $\cdot$ Service concepts

\section{Introduction}

Digitization is one of the most important trends of the 21 st century. The transformative force originating from digitization imposes a direct influence on value creation

The authors Valentin Holzwarth and Sebastian Steiner contributed equally to the work.

V. Holzwarth $(\varangle) \cdot$ J. Schneider $\cdot$ J. vom Brocke

Institute of Information Systems, University of Liechtenstein, 9490 Vaduz, Liechtenstein

e-mail: valentin.holzwarth@uni.li

V. Holzwarth

RhySearch, Network \& Innovation, 9471 Buchs, SG, Switzerland

S. Steiner · A. Kunz

ETH Zurich, Innovation Center Virtual Reality (ICVR), 8092 Zurich, Switzerland

(C) The Author(s), under exclusive license to Springer Nature Switzerland AG 2021 
in a corporate environment. In consequence, the current top five of the world's most valuable companies rely heavily on digital business models (Miller 2017). Also the Architecture, Engineering and Construction (AEC) sector is heavily affected by digitization, leading to an increased availability of data along project life-cycles and allowing for the creation of novel, smart services (Holzwarth et al. 2019).

However, the construction industry has not yet exploited the full potential of digitization (Gandhi et al. 2016). Schober and Hok (2016) identified four key factors to digital transformation in AEC: (i) Automation, (ii) Digital Data, (iii) Connectivity, and (iv) Digital Access. Mixed Reality (MR) is a technology, which supports the latter three of the four key factors to digital transformation in construction. It allows for an interaction with digital data, superimposed to a real environment. Thus, MR might enable the construction industry to further increase their degree of digitization and therefore improve its productivity. Prior research has already identified potential applications of MR in AEC (e.g., Wang et al. 2013). However, most prior works do not evaluate the potential of MR technology in a real context.

Therefore, the aim of the study presented in this paper is to explore the potentials of MR technology by conducting a case study in a representative real life setting. The case study's industry partner, one of the world's leading AEC suppliers, is seeking to develop MR-based services as a future complementary offering for their customers. This allows for an in-depth assessment of MR technology's potential in an industrial setting.

\section{Related Work}

This section provides an overview on the different applications for MR in the construction sector. The findings, where to apply MR technology to support construction processes, originate from different literature sources, such as the work done by Wang et al. (2013) on "a conceptual framework on integrating building information modelling with augmented reality". This section also serves as a basis to determine which MR-supported process should be examined during the user study.

MR technologies can be applied in the planning phase as well as in the construction phase (see Fig. 1). The planning phase includes all preliminary work such as the design and planning process, preparation of construction logistics, and any other preparation task. The construction phase describes the time during which the construction and quality control of the project takes place.

The applications of MR presented in this section are focusing on these planning and construction phase processes. Supporting other project life-cycle processes MR, such as tasks for facility management, is also possible and potentially promising. However, this is beyond the scope of this study and therefore not evaluated in more detail but should be mentioned for completeness. 


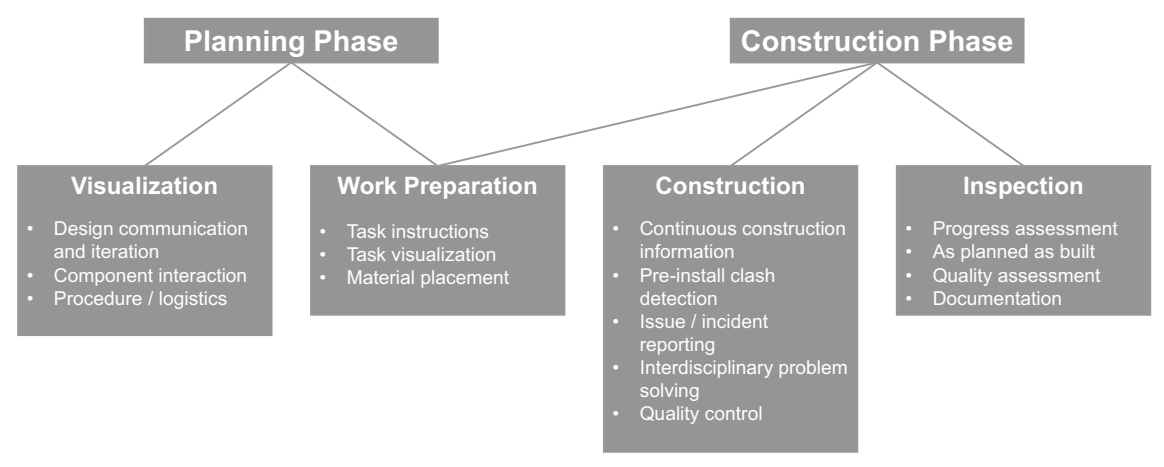

Fig. 1 Applications of MR in construction

\subsection{Visualization}

The application of MR technology allows for displaying plans and 3D modelsso-called Building Information Models (BIM) models-in the real world, either at their original size or at a smaller scale. This facilitates model comprehensibility for non-professionals and allows for visualizing substantially more information than conventional, printed plans. For example, with the MR platform CityScopeAR, developed by MIT media lab, collaborative urban design processes can be visualized. This system is used to display additional information about the implications that a planned project might have, such as congestion and construction costs (Noyman et al. 2019).

Another software solution for visualisation, which is available today, is the Trimble sketch up viewer which makes it possible to view the projects in a MR environment on a smartphone or with a Head-Mounted Display (HMD). Such software solutions allow the user to view the drawings as miniature 3D representations or in life-size, making them better comprehensible and the experience more immersive (Trimble 2019). Having such possibilities of visualizing plans with MR technology enables better understanding of component interaction, particularly between different planner professions, and it also enables a more interactive way of overall planning.

Additionally, construction logistics procedures can benefit from MR technology by connecting planners and the operating staff through simultaneous access to the same digital model. This enables fast response to unforeseen events or test runs of new logistics scenarios. Through these means, planners can be supported by operative staff during the planning process (Reif and Walch 2008).

Furthermore, MR technology is also considered to be valuable in supporting warehouse planning processes. MR technology can be used to visualize any planned rearrangement in full scale. This enables planners to not only test the plans on the real model but also to model new workflows, which are compatible with the changed environment (Glockner et al. 2014). 


\subsection{Work Preparation}

The second subcategory of the planning phase is work preparation, which is also linked to the construction phase, as planning is also required here. Besides the fact that task instructions and visualizations can be displayed before the task is carried out, other task-related information such as where to place material or machinery in order to achieve the best results can be communicated to the user as well. By that, a deeper connection between the early planning and the on-site preparation can be created, which allows for better accuracy and efficiency.

\subsection{Construction}

During construction, the execution of tasks plays a key role. The application of MR technology during task execution has been implemented at Boeing in the context of cable routing. Construction information is continuously made available to the user through an HMD, which provides technicians with a real-time, hands-free access to interactive 3D wiring diagrams. This MR-supported wiring process allows for more efficient task execution, which is consequently raising productivity. This has led to a $90 \%$ improvement in first-time quality compared to using two-dimensional information on the aeroplane, along with a $30 \%$ reduction in time spent on task execution (Boeing 2018). Besides the reported productivity improvements, MR technology leads to a reduced number of mistakes during complex assembly processes (Chalhoub and Ayer 2018).

\subsection{Inspection}

The final part of the construction phase is the inspection. Here, MR technology enables a facilitated and intuitive access to digital planning data directly on the job site. This enables progress and quality assessment by superimposing the real world with digital plans in an MR environment. The MR environment can be used to access information on the intended construction progress from digital plans more easily and thus enabling "as planned as built" comparisons. Also, the quality with which the features have been installed can be determined by comparing the location and alignment of the real world objects with their digital representations. These different inspection tasks set different requirements for the performance of the MR environment regarding accuracy and information allocation, with the latter being the most demanding for accuracy requirements. Since the user on the construction site has direct access to the digital data, accurate digital documentation of progress, quality and other performance indicators are possible through MR applications during inspection processes. This facilitates the collection and storage of digital data about 
various aspects of the construction process, which can lead to higher overall efficiency due to a higher data density. Utilizing MR technology in inspection tasks also enables self-inspection functionalities. This encourages job site workers to document their working processes and results on a regular basis. Furthermore, it prevents incorrect actions and helps workers to rectify errors immediately (Riexinger et al. 2018).

\section{Methodology}

The methodology of the study presented in this paper follows an exploratory, technology-driven approach, starting with an extensive review of MR applications in AEC, with the goal of creating service concept prototypes (see Fig. 2). The literature review summarizes both, the state-of-the-art in MR technologies, as well as prior research on MR applications in the context of AEC. This step results in a long-list of potential use cases. Subsequently, the technology selection evaluates the capabilities of hand-held and hands-free MR technology, regarding features such as calibration and accuracy. This step excludes those use cases that are not feasible due to technical constraints. A workshop with the industry partner adds specific requirements, which are combined with the technology selection's findings. Based on these steps, a user study is conducted, wherein 9 AEC professionals participate. In the first part of the user study, each participant is asked to solve a task supported by an MR application. In the second part of the user study, semi-structured interviews are conducted with each participant, which are transcribed and coded. In these semi-structured interviews, the participants contrast their expertise in AEC with the experience in solving the MR-supported task. Based on the user study's results, two MR-based service concept prototypes are developed: (i) a co-creation service, including a smartphone application to be utilized by involved stakeholders in the construction process, (ii) a full service, requiring only limited stakeholder interaction and collaboration.

Literature Review
Technology Selection

\section{Industry Requirements}

Fig. 2 The methodology of this paper 


\section{Results}

The results of the study in this paper follow the methodology's order, starting with the technology selection and concluding with the developed service concept prototypes.

\subsection{Technology Selection}

The technology selection identifies and analyzes two categories of MR technologies: (i) hand-held MR technology (e.g. smartphone MR applications) and (ii) handsfree MR technologies (e.g. HMDs). Accuracy (i.e. the offset of the superimposed virtual model over the real object) is essential for certain applications, such as quality assessment. A positional error (i.e. accuracy) of $6.64 \%$ at distances between 1.5 and $2.5 \mathrm{~m}$ has been reported for the Microsoft Hololens, which is a popular device for hands-free MR technology (Liu et al. 2018). For hand-held MR technology, no literature could be found. Thus, a benchmark experiment was performed, which yielded a $3.28 \%$ positional error at distances between 1.5 and $2.5 \mathrm{~m}$ for the iPhone $\mathrm{Xs}$, which is a popular representative of hands-free MR technology.

\subsection{User Study}

The user study integrates the findings of the literature review, technology selection and industry requirements of this study's partner company.

Materials The user study was conducted in a specifically prepared room at the industry partner's facility. For this room, a complete BIM model was available in the AEC software Autodesk Revit. ${ }^{1}$ Smartphone-based MR technology was chosen, due to accuracy concerns towards head-worn MR devices. The chosen setup was a state-of-the-art Apple iPhone Xs with the built-in ARKit technology and the MR application Gamma AR. ${ }^{2}$ For each participant, task completion time and the number of errors were recorded in the first part of the user study. In the second part of the user study, the participants reported on their experience in semi-structured interviews with a duration of approximately ten minutes. These interviews were recorded, transcribed and coded regarding usability and user acceptance criteria.

The Task During the user study, an inspection task is carried out with each participant in the following way: The participant is welcomed to the study session and informed about the inspection task. Furthermore, each participant is made aware of 5 defects in the study room, which are marked with a colored sticker (see Fig. 3). This was done in order to set the focus on the MR-assisted task and not on the identification

\footnotetext{
${ }^{1}$ https://www.autodesk.com/products/revit.

${ }^{2}$ https://gamma-ar.com/.
} 

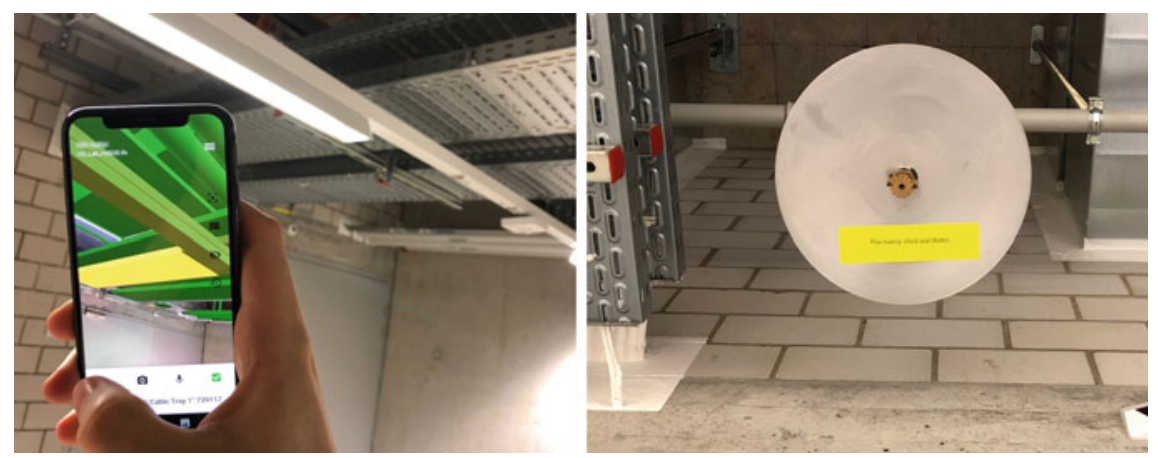

Fig. 3 The MR Application interface (left) and a defect marked with a colored sticker (right)

of defects. On each sticker, the type of the defect is noted. First, the participant has to set-up the MR environment by conducting a set-up calibration of the model. Then the participant uses the MR access to the BIM model of the study room to add the information which is stated on the coloured markers to the digital model (see Fig. 3). These colored markers define five possible defects or other issues that could potentially occur on a construction site. For each of the five defects, it is stated what the defect is and how the information should be added, either by a text, a photo, or an audio recording. This has to be done for all five defects.

This simplified user study setup can be imagined as part of a real world procedure, which can be divided into three parts: In part one, an inspector examines the construction site or specific parts of it in the "real world". When the inspector finds an error on a feature, has a general comment or wants to evaluate the progress, the MR environment provides access to the BIM model so that he can add or receive information.

In part two, there is no human interaction required. The comments made by the inspector are being uploaded to the cloud. Then the information can be automatically forwarded to the relevant professionals who oversee the specific parts. For example, if the inspector adds a comment on an electrical installation, the electrician will receive a notification and all the information about this installation. Also, all the comments are archived, which generates a timeline that might be used for performance evaluation.

In part three, the different technicians get a summary of the affected part (information available in the BIM model), where it is located and what the comments of the inspector are. With this information, they can collect the exact material they need to fix the issue. When arriving at the position which was marked in the ground plan of the building, the technician could again use the MR environment to locate the exact part. After fixing the issue, the MR environment is used again to add a comment to the part, whether the issue could be fixed or if there are any further actions required.

Participants Nine AEC professionals ( 6 male and 3 female) with a mean age of 27.56 $(\mathrm{SD}=6.82)$ years participated in the user study. They had various backgrounds, such as engineering, marketing, and computer science. All of the participants were daily 
smartphone users, whereas 5 participants had prior experience with MR technology. Only 3 participants had prior experience with BIM, while 5 participants were unfamiliar with 3D models of buildings.

After accomplishing the inspection task, all participants reported on their experience in semi-structured interviews. These interviews yielded that all 9 participants considered the MR application easy to use, 8 participants would like to use it frequently, and 7 participants stated it would make their work more enjoyable. Furthermore, 2 participants saw the main benefit in process transparency (i.e. cover-ups for defects are impeded due to fast and transparent issue identification processes).

\subsection{Service Concept Prototypes}

Based on the positive findings of the user study regarding performance, user acceptance, and usability, two prototypes for a MR-based service supporting inspection tasks on construction sites were envisioned:

Co-creation service The service would be provided in form of a digital platform, consisting of a smartphone MR application and a cloud-based issue management software. This service requires value co-creation of all workers and inspectors on a construction site (i.e. the value is created by all involved actors together, instead of one actor creating and the other actors consuming the value). Although the findings of the user study were very positive, it is still doubted, whether all stakeholders would engage in the service. This could not only be due to resistance towards novel technology, but also due to transparency that would impede cover-ups of construction defects.

Full service The full service relies on the same infrastructure as the co-creation service. Here, the service does not require any co-creation. This is achieved by dedicated teams, managing issues and defects on construction sites. This service concept eliminates the disadvantage of the co-creation service, as it does not require the participation of construction site stakeholders. However, the full service would be substantially more costly for the construction project and would be only chosen for special projects.

\section{Discussion}

The overview of Wang et al. (2013) on applications of MR in AEC served as the basis for this paper. However, it has proven to be challenging to develop a use case that fulfills industry requirements, is technically feasible, and has potential for substantial improvements due to MR support. The inspection of building defects with smartphone-based MR is such a use case. It took the participants on average three minutes and $30 \mathrm{~s}$ to complete the task, this is equal to about $40 \mathrm{~s}$ per identified issue. 
The time was measured including the calibration time but excluding the time it took to restart the application if this was necessary during the user study. Overall, it took the participants 1.67 calibration attempts to set-up the MR environment correctly. This means that $40 \%$ of calibration attempts were unsuccessful. Surprisingly, participants were not heavily bothered by unsuccessful calibration and reported high usability and acceptance. However, the accuracy (i.e. the offset of the virtual model) was an issue for 3 participants.

\section{Conclusion and Outlook}

The aim of this study - exploring the potentials of MR in AEC — was fulfilled. The first substantial finding is that today's technology already seems to be suited to support some of the basic tasks on a construction site. This is most importantly supported by the participants of the user study, who stated that they could even imagine using the MR setup in its current form. Additionally, it is important to note that a regular smartphone could support the MR application which guarantees a high degree of accessibility. The most meaningful finding, however, is not only that this technology is sufficiently mature and accessible but that it is genuinely considered an improvement over a non-MR-supported workflow. Some participants considered the improvement to become apparent during the process itself, but all agreed that the higher quality of data, which was available after using this technology, as well as the time savings due to much faster data post-processing, are undoubtedly the substantial benefits.

Despite these promising results, some problems were also identified. Firstly, it became clear that the calibration success rate has to be improved in order to accelerate the workflow. Secondly, the overall accuracy of the software needs to be improved in order to make more detail-oriented tasks possible and to avoid generating the necessity for users to compensate for software flaws. Further research should be considered regarding the user acceptance on every level of construction work since the scope of our study could not sufficiently cover such a large spectrum. Therefore, conducting experiments with more participants from different backgrounds in the construction industry and directly comparing MR processes with the currently utilized processes on the construction site as well as comparing different MR solutions would be a consequent development of the presented study.

When discussing the limitations of MR technologies, one also has to consider the technology's potential. Current issues pale in comparison to the manifold future possibilities of developing MR technology further. The great variety of possible future applications was demonstrated by the participants immediately being able to think of further tasks on a construction site, which could be supported by MR. All these tasks could consequently lead to a fully MR-supported construction process. In this process, every participating worker could stay well informed through the data 
provided by such an MR system. In this sense, MR would serve as the key technology to a full digitization of construction sites, possibly introducing one of the greatest efficiency improvements of recent times.

Acknowledgements This work was supported by a project financed by the Hilti Family Foundation in Schaan, Liechtenstein and RhySearch in Buchs SG, Switzerland. Additionally, the authors would like to thank Hilti AG for the fruitful collaboration.

\section{References}

Boeing. (2018). Boeing tests augmented reality in the factory. Retrieved from https://www.boeing. com/features/2018/01/augmented-reality-01-18.page.

Chalhoub, J., \& Ayer, S. K. (2018). Using mixed reality for electrical construction design communication. Automation in Construction, 86, 1-10. https://doi.org/10.1016/j.autcon.2017.10.028.

Gandhi, P., Khanna, S., \& Ramaswamy, S. (2016). Which industries are the most digital (and why)? Harvard Business Publishing. Retrieved from https://hbr.org/2016/04/a-chart-that-showswhich-industries-are-the-most-digital-and-why.

Glockner, H., Jannek, K., Mahn, J., \& Theis, B. (2014). Augmented reality in logistics. DHL Customer Solutions \& Innovation. Retrieved from https://www.dhl.com/content/dam/downloads/g0/ aboutus/logisticsinsights/csiaugmentedrealityreport290414.pdf.

Holzwarth, V., Schneider, J., Kunz, A., \& vom Brocke, J. (2019). Approaching data driven services in the built environment. In J. Meierhofer, \& S. West (Eds.), 2nd smart services summit. Swiss Alliance for Data-Intensive Services.

Liu, Y., Dong, H., Zhang, L., \& Saddik, A. E. (2018). Technical evaluation of HoloLens for multimedia: A first look. IEEE Multi-Media, 25(4), 8-18. https://doi.org/10.1109/mmul.2018.2873473.

Miller, L. J. (2017). Ten years of iphones have made apple the world's no. 1 company. Retrieved from https://www.bloomberg.com/news/articles/2017-09-11/apple-vaults-to-no-1from-no-70-after-a-decade-of-iphone-sales.

Noyman, A., Sakai, Y., \& Larson, K. (2019). Cityscopear: Urban design and crowdsourced engagement platform.

Reif, R., \& Walch, D. (2008). Augmented \& virtual reality applications in the field of logistics. The Visual Computer, 24(11), 987-994. https://doi.org/10.1007/s00371-008-0271-7.

Riexinger, G., Kluth, A., Olbrich, M., Braun, J.-D., \& Bauernhansl, T. (2018). Mixed reality for on-site self-instruction and self-inspection with building information models. Procedia CIRP, 72, 1124-1129. https://doi.org/10.1016/j.procir.2018.03.160.

Schober, K.-S., \& Ho, P. (2016). Digitization in the construction industry. Roland Berger GmbH. Retrieved from https://www.rolandberger.com/publications/publicationpdf/ tabdigitizationconstructionindustryefinal.pdf.

Trimble. (2019). Trimble sketchup viewer. Retrieved from https://www.sketchup.com/products/ sketchup-viewer.

Wang, X., Love, P. E., Kim, M. J., Park, C.-S., Sing, C.-P., \& Hou, L. (2013). A conceptual framework for integrating building information modeling with augmented reality. Automation in Construction, 34, 37-44. https://doi.org/10.1016/j.autcon.2012.10.012. 